\title{
Prevalence of Psychiatric Diagnoses in Pediatric Chiari Malformation Type 1
}

\author{
Maureen Lacy ${ }^{a}$ Samantha DeDios-Stern ${ }^{a}$ Sarah Fredrickson ${ }^{a}$ Shravan Parikh ${ }^{a}$ \\ Todd Nader ${ }^{\mathrm{a}}$ David M. Frim ${ }^{\mathrm{b}}$ \\ ${ }^{a}$ Department of Psychiatry and Behavioral Neuroscience, The University of Chicago, Chicago, IL, USA; bection of \\ Neurosurgery, The University of Chicago, Chicago, IL, USA
}

\section{Keywords}

Chiari malformation · Psychiatry · Depression · Anxiety ·

Attention deficit hyperactivity disorder

\begin{abstract}
This cross-sectional study investigates the prevalence and risks for psychiatric diagnoses in a large cohort of children with Chiari malformation type 1 (CM1) presenting for neurosurgical evaluation. Children between the ages of 6 and 17 years who were evaluated and diagnosed with CM1 at a neurosurgery clinic were identified. Eighty-six participants were recruited for this study with an average age of 11 years. Parents of participants completed a pediatric medical history questionnaire and a semistructured interview regarding the child's psychiatric, developmental, medical, and family history. A review of medical records was completed to complement interview data. Elevated rates of psychiatric diagnoses, including attention deficit hyperactivity disorder (ADHD) (22.1\%), anxiety (12.8\%), and depression (10.5\%), were identified in the study population when compared to published norms in the general population documented by the American Psychiatric Association in 2013. In addition, elevated rates of psychiatric diagnoses in first-degree relatives of
\end{abstract}

\section{KARGER}

() 2018 S. Karger AG, Basel

E-Mail karger@karger.com

www.karger.com/pne study participants were also identified. A 2-step binary logistic regression analysis revealed that maternal complications during pregnancy (Wald $=6.52, p=0.01$ ) increased the risk of a psychiatric diagnosis 9-fold. Premature birth (Wald = $6.79, p=0.01$ ) also significantly predicted a psychiatric diagnosis amongst participants. The current findings suggest a high prevalence of psychiatric illness in children with CM1. Pregnancy complications were associated with a high risk of a psychiatric diagnosis. Early CNS developmental disturbance may explain this relationship. Prematurity only slightly improved the prediction model. Limitations and future directions are discussed, including the cross-sectional nature of the present study, possible self-selection bias, and the importance of future investigation of other causative and/or associative factors of $\mathrm{CM} 1$, such as cognitive, psychiatric, and medical influences on health status. @ $2018 \mathrm{~S}$. Karger AG, Basel

\section{Introduction}

Chiari malformation type 1 (CM1) is viewed as a congenital hindbrain aberration typically characterized by hypoplastic posterior fossa, disruption of cerebrospinal 
fluid dynamics, and downward displacement of the cerebellar tonsils. Cerebellar tonsillar herniation $5 \mathrm{~mm}$ below the foramen magnum has historically been utilized as a radiological diagnostic indicator, although this criterion has been debated in the literature over the years [1-3]. Imaging-based prevalence studies estimate CM1 as occurring in approximately $0.24-3.6 \%$ of the population [4]. Clinical findings are varied and often include tussive headache, dizziness, dysphagia, extremity or body pain, and nystagmus [5]. MR studies have noted diffusion imaging abnormalities in the middle cerebellar peduncle in symptomatic patients $[6,7]$ though the etiology of many of the symptoms associated with CM1 is incompletely understood. Common syndromes also associated with CM1 include hydrocephalus, pseudotumor cerebri, and syringomyelia $[8,9]$. Diagnosis is often based on symptom presentation coupled with imaging findings. Treatment options include observation or decompressive surgery. CM1 is considered primarily sporadic, although recent studies of familial clustering have indicated a possible genetic component [2].

Research studies attempting to define CM1 often address physical symptoms, medical comorbidities, and surgical outcomes $[2,8,9]$, with few studies examining psychiatric factors. At present, there are only a few case studies and online-based surveys examining psychiatric diagnoses associated with CM1. In a recent study, Bakim et al. [10] documented a high incidence of psychiatric disorders in a small sample of postsurgical adults with CM1. Also, a recent online survey targeting adults with CM1 revealed a high rate of self-reported mood disorders [11]. Additionally, published case reports have documented anxiety disorders [12-14] and psychosis associated with CM1 [15]. Finally, a published case series has vaguely referenced "mental retardation and behavioral autistic type" in a cohort of children with CM1 [16].

This gap in the literature is surprising as cerebellar networks have been implicated as playing a significant role in psychiatric disorders. Schmahmann and Sherman [17] first documented executive and emotional dysregulation in a cohort of patients with cerebellar insults and coined the term cerebellar-cognitive affective syndrome. Over the last 2 decades, structural and functional abnormalities of the cerebellum and cerebellar networks have been associated with a wide range of psychiatric disorders, including ADHD, depression, anxiety, autism, schizophrenia, and bipolar disorders [18].

The objective of this investigation was to determine the prevalence of psychiatric diagnoses among a large cohort of children formally diagnosed with CM1. As many of these children have comorbid conditions (e.g., prematurity [19] and epilepsy [20]) associated with psychiatric disorders, we examined the contributing impact of medical comorbidities on psychiatric risk.

\section{Methods}

This cross-sectional study was approved by the University of Chicago Medicine Institutional Review Board and followed Helsinki Guidelines. A total of 86 individuals provided data for this study; they represented a convenience sample collected from all eligible patients presenting over a 5-year period for evaluation of CM1 at a university pediatric neurosurgery clinic, and their families agreed to participation with patient assent. Families were eligible to participate if their child with CM1 was between the ages of 6 years, 0 months and 17 years, 11 months, and if their CM1 diagnosis was confirmed by the senior author. After obtaining parent and child consent, parents of participants completed a pediatricbased medical and psychiatric history questionnaire. All parents and children then completed a semistructured interview about the child's psychiatric, developmental, medical, and family histories. A review of the medical record was also completed to complement the history. Either a licensed clinical neuropsychologist or a trained graduate-level research assistant under direct supervision of the same licensed clinical neuropsychologist conducted the interview and medical record review.

\section{Data Analyses}

Means, standard deviations, and frequencies were calculated for the participant and family demographic statistics. Frequencies were calculated for all developmental, medical, and psychiatric history variables, as well as immediate family psychiatric history. Finally, a two-step binary logistic regression analysis was conducted to investigate variables which may predict whether participants have a psychiatric history. In the first step of the logistic regression, all developmental and medical variables were entered as predictors, with family psychiatric history entered in the second step. For all statistical analyses, significance was indicated if $p$ was less than 0.05 .

\section{Results}

\section{Participants}

Demographic statistics for the sample are presented in Table 1. The sample consisted of 44 males and 42 females with a mean age of 11 years, 3 months ( $\mathrm{SD}=3$ years, 5 months). Most participants were right-handed (87.2\%), Caucasian $(81.4 \%)$, and diagnosed with CM1 at a mean age of 7 years, 2 months (SD $=4$ years, 6 months). The majority of participants' families had an estimated annual household income USD $>74,000$ per year (51.2\%), with the majority of mothers and fathers having at least 12 years of education ( $84.8 \%$ of mothers and $80.2 \%$ of fa- 
Table 1. Children $(n)$ with Chiari malformation type 1: demographic statistics $(n=86)$

\begin{tabular}{|c|c|}
\hline Mean age (SD), years & $11.3(3.5)$ \\
\hline At Chiari diagnosis & $7.2(4.6)$ \\
\hline \multicolumn{2}{|l|}{ Sex } \\
\hline Male & $44(51.2 \%)$ \\
\hline Female & $42(48.8 \%)$ \\
\hline \multicolumn{2}{|l|}{ Handedness } \\
\hline Right & $75(87.2 \%)$ \\
\hline Left & $9(10.5 \%)$ \\
\hline Ambidextrous & $2(2.3 \%)$ \\
\hline \multicolumn{2}{|l|}{ Race } \\
\hline Caucasian & $70(81.4 \%)$ \\
\hline African American & $4(4.7 \%)$ \\
\hline Hispanic & $4(4.7 \%)$ \\
\hline Asian & $1(1.2 \%)$ \\
\hline Multiracial & $6(7.0 \%)$ \\
\hline Unknown & $1(1.2 \%)$ \\
\hline \multicolumn{2}{|l|}{ Est. annual household income } \\
\hline USD $<35,000$ & $1(1.2 \%)$ \\
\hline USD 35,000-41,999 & $4(4.7 \%)$ \\
\hline USD $42,000-51,999$ & $8(9.3 \%)$ \\
\hline USD 52,000-58,999 & $8(8.3 \%)$ \\
\hline USD 59,000-73,999 & $19(22.1 \%)$ \\
\hline $\mathrm{USD} \geq 74,000$ & $44(51.2 \%)$ \\
\hline Unknown & $2(2.3 \%)$ \\
\hline \multicolumn{2}{|l|}{ Mothers' years of education } \\
\hline $0-7$ years & $0(0.0 \%)$ \\
\hline 8 years & $0(0.0 \%)$ \\
\hline $9-11$ years & $2(2.3 \%)$ \\
\hline 12 years & $15(17.4 \%)$ \\
\hline $13-15$ years & $19(22.1 \%)$ \\
\hline$\geq 16$ years & $39(45.3 \%)$ \\
\hline Unknown & $3(3.5 \%)$ \\
\hline \multicolumn{2}{|l|}{ Fathers' years of education } \\
\hline $0-7$ years & $0(0.0 \%)$ \\
\hline 8 years & $0(0.0 \%)$ \\
\hline $9-11$ years & $2(2.3 \%)$ \\
\hline 12 years & $15(17.4 \%)$ \\
\hline $13-15$ years & $16(18.6 \%)$ \\
\hline$\geq 16$ years & $38(44.2 \%)$ \\
\hline Unknown & $6(7.1 \%)$ \\
\hline \multicolumn{2}{|l|}{ Decompression surgery status } \\
\hline Underwent surgery & $43(50 \%)$ \\
\hline Did not undergo surgery & $43(50 \%)$ \\
\hline
\end{tabular}

thers). Investigation of decompression surgery status revealed that $50 \%(n=43)$ had undergone decompression surgery.

\section{Medical Comorbidities}

Frequencies of medical conditions common among CM1 patients were characterized for the sample and are presented in Table 2. Regarding development, $43.0 \%$ of
Table 2. Medical comorbidities $(n=86)$

\begin{tabular}{ll}
\hline Pregnancy/delivery complications & $43 \%$ \\
Premature birth status & $26.7 \%$ \\
Developmental delay & $32.6 \%$ \\
Neurological conditions & \\
$\quad$ Syringomyelia & $16.3 \%$ \\
Seizures & $15.1 \%$ \\
Pseudotumor cerebri & $11.6 \%$ \\
Head injury & $10.5 \%$ \\
Tethered cord & $5.8 \%$ \\
Hydrocephalus & $8.1 \%$ \\
\hline
\end{tabular}

participants' parents noted some problem during pregnancy or birth of the participant, such as nuchal cord, preeclampsia, and/or abnormal biophysical profile on ultrasound. Additionally, $26.7 \%$ of participants were reported as being born premature, defined as birth before 37 weeks of pregnancy, and $32.6 \%$ of participants were characterized as developmentally delayed. Neurologically, participants also experienced a range of conditions, with syringomyelia $(16.3 \%)$, seizures $(15.1 \%)$, and pseudotumor cerebri (11.6\%) being the most commonly endorsed medical conditions. Conversely, head injury (10.5\%), tethered cord (5.8\%), and hydrocephalus (8.1\%) were less commonly reported conditions.

\section{Child Psychiatric History}

Regarding the participants' psychiatric history, approximately half (53.5\%) reported no history of a psychiatric diagnosis. The frequencies of participant psychiatric diagnoses are characterized in Figure 1. Overall, ADHD was the most common psychiatric condition (22.1\%), followed by anxiety (12.8\%) and depression (10.5\%). Parents less commonly endorsed a history of bipolar disorder $(2.3 \%)$ or autistic spectrum disorder (3.5\%) for their child.

\section{Family Psychiatric History}

Frequencies of reported family psychiatric conditions are displayed in Figure 2. Psychiatric diagnoses were reported only for immediate family members (biological parents or biological siblings). Overall, $55.8 \%$ of participants had no significant family psychiatric history; however, many participants' had immediate family members with a history of anxiety (30.2\%), ADHD (25.6\%), and/or depression (23.3\%). No family members had a history of schizophrenia ( $0 \%)$, with over $3 \%$ diagnosed with bipolar disorder (3.5\%) and autism 


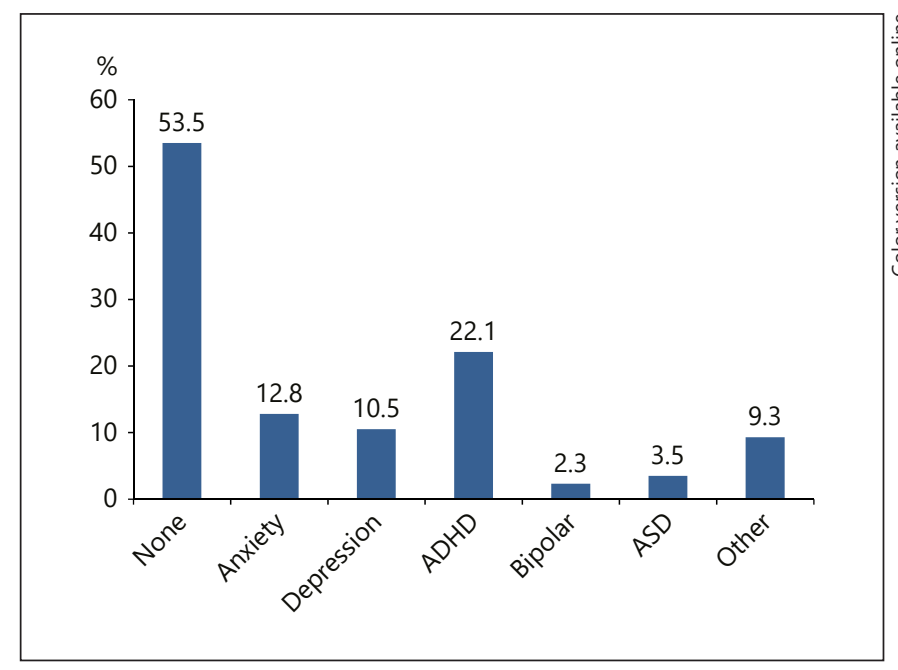

Fig. 1. Percentage of participants who reported the above psychiatric histories. ADHD, attention deficit hyperactivity disorder; ASD, autism spectrum disorder.

Table 3. Results of the 2-step logistic regression $(n=70)$

\begin{tabular}{lcccc}
\hline Variable & $\chi^{2}$ & Wald & $p$ & OR \\
\hline Step 1 & & & & \\
Developmental/medical var.** & 26.65 & & 0.003 & \\
$\quad$ Decompression surgery & & 0.01 & 0.92 & 0.94 \\
$\quad$ Prematurity** & 6.79 & 0.01 & 0.08 \\
$\quad$ Developmental delay & 1.28 & 0.26 & 2.23 \\
$\quad$ Problems in pregnancy** & 6.52 & 0.01 & 8.69 \\
$\quad$ Seizures & 3.64 & 0.06 & 0.14 \\
$\quad$ Hydrocephalus & 0.45 & 0.50 & 0.44 \\
$\quad$ Pseudotumor cerebri & & 1.09 & 0.30 & 2.98 \\
$\quad$ Syrinx/syringomyelia & & 2.43 & 0.12 & 4.35 \\
$\quad$ Tethered cord & & 0.00 & 1.00 & 0.00 \\
$\quad$ Head injury & 0.71 & 0.40 & 0.36 \\
Step 2 & & & \\
Family psychiatric history & 2.24 & & 0.13 & \\
$\quad$ Present & & 2.19 & 0.17 & 2.65 \\
\hline * $p<0.05,{ }^{* *} p<0.01$. OR, odds ratio. & & & \\
\hline
\end{tabular}

spectrum disorder (3.5\%). Approximately $4.7 \%$ of participants' family members had other types of psychiatric conditions not listed, such as Tourette's syndrome and substance abuse disorders. Of note, 1 (1.2\%) individual's family psychiatric history was unknown as that individual was adopted.

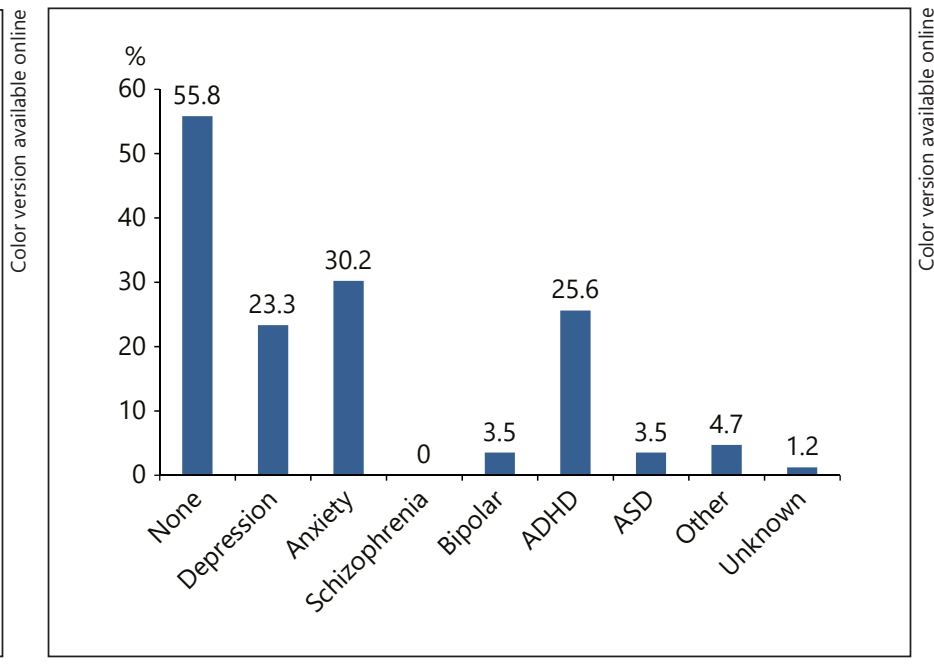

Fig. 2. Percentage of the participants who reported family a psychiatric history among immediate family members (mother, father, or siblings). ADHD, attention deficit hyperactivity disorder; $\mathrm{ASD}$, autism spectrum disorder.

\section{Analysis of Psychiatric History and Medical Comorbidities}

A follow-up 2-step binary logistic regression analysis was conducted to investigate possible predictors of psychiatric history in the present CM1 pediatric sample (Table 3). Complete data for all of the study variables of interest were available for 70 of the 86 participants; therefore, listwise deletion was used to treat the missing data, and only these individuals were included in the logistic regression analysis. Prior to entering any variables into the regression model, whether or not participants had a psychiatric history was estimated to be accurately predicted $54.3 \%$ of the time. In the first step, decompression surgery status, developmental variables, and medical variables for the participants were entered, resulting in a significant improvement in the accuracy of the model prediction to $70.0 \%, \chi^{2}=26.65, \mathrm{df}=10, p<0.01$, HosmerLemeshow test, $p=0.87$. Overall, being born premature (Wald $=6.79, p=0.01$ ) and a history of complications during pregnancy (Wald $=6.52, p=0.01$ ) significantly predicted psychiatric history among participants. Having a history of seizures was approaching significance as a predictor of psychiatric history (Wald $=3.64, p=0.06$ ). When participants were either born premature or had a history of seizures, they were marginally more likely to have a psychiatric history ( 0.08 times and 0.14 more likely, respectively). Notably, when participants' gestation or birth was complicated, the participant was 8.86 times 
more likely to have a psychiatric diagnosis. No other medical or developmental variables were significant predictors of psychiatric history. In the second step, family psychiatric history was entered as a predictor; however, inclusion of this variable only improved the ability of the model to predict participant psychiatric history by approximately $4 \%$ ( $74.3 \%$ total), which was not a statistically significant improvement, $\chi^{2}=2.24, \mathrm{df}=1, p=0.14$, Hosmer-Lemeshow test, $p=0.91$. Therefore, only prematurity status and complications during pregnancy significantly predicted presence of participant psychiatric history.

\section{Discussion}

The objective of this study was to investigate the prevalence and risks for psychiatric illnesses in a large cohort of children with CM1. A high rate of psychiatric disturbance was predicted based on review of the literature, a high rate of comorbid risk factors, and cerebellar involvement. Study findings indicated almost 1 out of 2 (47\%) children with CM1 reported having a psychiatric diagnosis. This is notable given that the average age of the cohort was only 11 years. A recent meta-analysis [21] indicated a worldwide prevalence rate of $13.4 \%$ for any mental disorder for children and adolescents. More specifically, the worldwide prevalence for depressive disorders was $2.6 \%$, anxiety disorders $6.5 \%$, and $\mathrm{ADHD} 3.4 \%$. In the current cohort, ADHD, anxiety, and depressive disorders were the most commonly reported psychiatric conditions, with rates between 10 and $22 \%$. Additionally, rates of autism spectrum disorders (3.5\%) and bipolar disorders $(2.3 \%)$ in our pediatric sample were greater than national prevalence rates that approach $1 \%$ for both disorders [22]. The recent Conquer Chiari online survey of adults with CM1 [11] revealed that about one-third of adults with CM1 reported suffering from depression, and 20\% endorsed clinical anxiety. While our findings are based on family report and record review, these rates are consistent with the study by Bakim et al. [10], who documented a $44 \%$ rate of psychiatric disorders in an adult CM1 sample using a standardized clinical interview format.

In addition to elevated rates of psychiatric diagnoses in children with CM1 in the current sample, the present study also documented a high rate of family psychiatric illness. Similar to the prevalence rates for the CM1 children, results revealed that anxiety (30\%), ADHD (26\%), and depression $(23 \%)$ were the most prevalent psychiatric disorders reported within families. While parental psy-

Psychiatric Diagnoses in Chiari

Malformation 1 chiatric illness has been found to be a risk factor for childhood psychiatric illness in previous studies [23], elevated rates of family psychiatric diagnoses did not appear to add greatly to the prediction model of childhood psychiatric illnesses in the current study.

In regard to family demographics, it is worth noting that this was one of the first studies to document sociodemographic variables, including race, family income, and parents' level of education in a cohort of CM1 children. Specifically, the sample was overwhelmingly Caucasian (81\%), and approximately $51 \%$ of participants' families had an estimated annual household income USD $>74,000$ per year. Moreover, the majority of mothers and fathers had at least 12 years of education ( $84.8 \%$ of mothers and $80.2 \%$ of fathers). These findings may represent cultural influences on help-seeking behavior and/or access to care in regard to specialized services (e.g., neurosurgery consultation). Given the high degree of individual and family psychiatric history reported, it is also possible that these individuals were more inclined to seek out services due to prior experience with psychiatric treatment. Many studies examining surgical outcomes before and after decompression often underappreciate the significance of these family sociodemographic characteristics as factors that could potentially impact access to and quality of care. More studies in the future should include these factors to further aid in this discussion.

An understanding of the link between CM1 and childhood-onset psychiatric illness remains elusive. Interestingly, a history of complications during pregnancy was found to be the greatest risk of having a psychiatric history. Specifically, individuals who had suffered some form of complication during pregnancy were almost 8 times more likely to have a psychiatric disorder than those children who did not experience complications during pregnancy. Prematurity added slightly to the predictive model as did a history of epilepsy. It has been suggested that early disruption of the developing neonatal nervous system has been associated with later psychiatric problems [2426]. Nosarti et al. [25] hypothesized that the immature brain is vulnerable to neonatal injury, which may lead to psychiatric problems later in life via faulty corticogenesis. In addition, disruptions of neuroanatomical networks, such as frontoparietal-cerebellar connections, have been observed in individuals born preterm that are similar to changes seen in psychiatric populations. An increasing number of $3 \mathrm{D}$ volumetric MRI studies continues to demonstrate a critical period of cerebellar development taking place in the third trimester, at which time the cerebellum undergoes its most rapid growth (unlike any other cere- 
bral structure) [27]. Studies have suggested that prenatal cerebellar disruptions can lead to a heterogeneous presentation of psychiatric disorders. This is in part due to the timing and chronicity of injury in relation to the stage of cerebellar development [28]. The lack of specificity in connecting specific psychiatric diagnoses with neurodevelopmental abnormalities indicates similar neurodevelopmental etiologies or common pathways that give rise to a full spectrum of psychiatric outcomes [29]. Interesting, yet preliminary, a recent diffusion tensor imaging study [6] implicated disrupted white matter tracks, specifically in the middle cerebellar peduncles, in children with CM1. Notably, white matter integrity has been associated with a wide range of psychiatric disorders, including depression, $\mathrm{ADHD}$, and anxiety [29].

Other hypotheses that attempt to understand the underlying mechanism of psychiatric morbidity in CM1 have been posited. Specifically, Schmahmann and Sherman [17] suggested a topographic organization of the cerebellum, whereby specific cerebellar lesions and abnormalities affecting primarily the posterior region and vermis have resulted in the cerebellar cognitive affective syndrome. Although CM1 does not directly involve the posterior region of the cerebellum, various subcortical networks including afferent pathways involving the middle cerebral peduncles, disruption in CSF flow dynamics, and increased intracranial pressure can potentially result in symptomatic complaints that may appear to be less severe and distressing as opposed to an overt cerebellar cognitive affective syndrome type of presentation. Moreover, Caykoylu et al. [12] hypothesized that the co-occurrence of CM1 and psychiatric conditions may be attributed to compression of the brainstem circuitry by CM1 leading to disruption of key neurotransmitters involved in mood state, especially serotonergic and noradrenergic systems. While a recent diffusion tensor imaging study in a small cohort failed to find any white matter abnormalities in the lower brain stem [6], based on a high rate of comorbid sleep and heart rate issues, ongoing investigation with larger samples is warranted. Moreover, the hypothesis that decompressive Chiari surgery may cause or exacerbate comorbid psychiatric illness is of surgical interest. In an adult sample, Allen et al. [30] suggested that neurocognitive symptoms might be attributed to surgical insult associated with decompression injury. However, psychiatric indicators such as depression or anxiety were not noted. If, in fact, this proves to be true, methods to diagnose and determine susceptibility to surgical exacerbations of baseline psychiatric disease in pediatric $\mathrm{CM} 1$ patients will need to be explored.

\section{Limitations and Future Directions}

While the present study represents the first attempt to examine the psychiatric history of children formally diagnosed with CM1 in the largest published cohort to date, several limitations on interpretation and thoughts on future directions are warranted at this time. First, psychiatric history was based on medical record review and parent report through semistructured interviews. Future studies should include formalized clinical interviewing, such as the Structured Interview for Diagnostic and Statistical Manual (DSM) Disorders (SCID), along with more standardized psychiatric assessment measures to ascertain formalized diagnosis in line with the DSM-5, and distinguish whether or not individuals are presently symptomatic or asymptomatic. Second, there is an inherent sampling bias that is unavoidable in many respects. This sample includes symptomatic children seeking formal neurosurgical consultation and thus may not accurately generalize to the larger population of individuals who are diagnosed with CM1, many of whom are asymptomatic. A sample of convenience was utilized, and thus it is possible that individuals with a psychiatric history or symptomatic complaints sought out services, while individuals with no history declined additional services. Although this may represent a selection bias in terms of individuals wanting to seek out care, this also reflects the nature of symptomatic versus asymptomatic features of CM1 and the propensity of the former group to seek out clinical care at a neurosurgery clinic. In order to bolster our findings, the use of a control group would have been beneficial; however, given that this study aimed to document psychiatric risk factors within a CM1 sample, future studies can disentangle the causative and/or associative factors of CM1, including possible cognitive, psychiatric, and medical influences on health status. Despite limitations concerning a selection bias, this study was able to document a significant amount of family sociodemographic information that is otherwise often overlooked in CM1 studies. The information gleaned from the subsequent data can help health care providers understand who is more likely to seek out services in the context of surgical consultation while developing efforts to target low socioeconomic status and culturally diverse populations. Third, no imaging data were obtained for this study. Future studies would be strengthened by examining the relationship between psychiatric presentation and CNS variables, such as level of herniation, integrity of cerebral white matter tracks, cerebrospinal fluid dynamics, intracranial pressure, and posterior fossa volume. This may lead to a better understanding of the etiology of our find- 
ings. Fourth, further information regarding perinatal complications and parent medical and substance abuse histories is warranted to gain a better understanding of these findings. Fifth, as this study was cross-sectional, the present data represent only one time point. It will be important for future studies to collect longitudinal data regarding medical conditions, as well as physical and psychiatric symptoms among individuals with CM1 in order to better understand if these relationships between the study variables may change over time, and whether psychiatric symptoms may be impacted by neurological symptoms. Finally, the relationship between psychiatric illness and common copresenting symptoms (e.g., headaches and sleep disorders) may help elucidate an underlying etiology.

\section{Conclusions}

Overall, this is the first and largest study to date that systematically examines the prevalence of psychiatric diagnoses in children diagnosed with CM1. Specifically, the current study documented elevated rates of depression, anxiety, and ADHD compared to healthy peers. Risks for psychiatric diagnoses in this sample included complications during pregnancy and to a lesser extent prematurity and seizure history. Future research should seek to explore biopsychosocial factors related to CM1 that may contribute to psychiatric outcomes. The current findings also begin to ask whether screening for psychiatric illness in the CM1 population may contribute to treatment outcomes including the efficacy of surgical decompression.

\section{Acknowledgments}

This study was funded by a Conquer Chiari Foundation and Column of Hope Research grant, as well as NIH grant R01NS41440-01 to D.M.F.

\section{Disclosure Statement}

The authors report no conflict of interest concerning the materials or methods used in this study or the findings specified in this paper.

\section{References}

1 Milhorat TH, Chou MW, Trinidad EM, Kula RW, Mandell M, Wolpert C, Speer MC: Chiari I malformation redefined: clinical and radiographic findings for 364 symptomatic patients. Neurosurgery 1999;44:1005-1017.

2 Arnautovic A, Splavski B, Boop FA, Arnautovic KI: Pediatric and adult Chiari malformation type I surgical series 1965-2013: a review of demographics, operative treatment, and outcomes. J Neurosurg Pediatr 2015;15:161177.

3 Greenberg JK, Yarbrough CK, Radmanesh A, Godzik J, Yu M, Jeffe DB, et al: The Chiari Severity Index: a preoperative grading system for Chiari malformation type 1 . Neurosurgery 2015;76:279-285.

4 Kahn EN, Muraszko KM, Maher CO: Prevalence of Chiari I malformation and syringomyelia. Neurosurg Clin N Am 2015;26:501507.

5 Durham SR, Fjeld-Olenec K: Comparison of posterior fossa decompression with and without duraplasty for the surgical treatment of Chiari malformation type I in pediatric patients: a meta-analysis. J Neurosurg Pediatr 2008;2:42-49.

6 Eshetu T, Meoded A, Jallo GI, Carson BS, Huisman TA, Poretti A: Diffusion tensor imaging in pediatric Chiari type I malformation. Dev Med Child Neurol 2014;56:742-748.
7 Kumar M, Rathore RK, Srivastava A, Yadav SK, Behari S, Gupta RK: Correlation of diffusion tensor imaging metrics with neurocognitive function in Chiari I malformation. World Neurosurg 2011;76:189-194.

8 Tubbs RS, Beckman J, Naftel RP, Chern JJ, Wellons JC 3rd, Rozzelle CJ, et al: Institutional experience with 500 cases of surgically treated pediatric Chiari malformation type I. J Neurosurg Pediatr 2011;7:248-256.

9 Fagan LH, Ferguson S, Yassari R, Frim DM: The Chiari pseudotumor cerebri syndrome: symptom recurrence after decompressive surgery for Chiari malformation type I. Pediatr Neurosurg 2006;42:14-19.

10 Bakim B, Goksan Yavuz B, Yilmaz A, Karamustafalioglu O, Akbiyik M, Yayla S, et al: The quality of life and psychiatric morbidity in patients operated for Arnold-Chiari malformation type I. Int J Psychiatry Clin Pract 2013;17:259-263.

11 Fischbein R, Saling JR, Marty P, Kropp D, Meeker J, Amerine J, et al: Patient-reported Chiari malformation type I symptoms and diagnostic experiences: a report from the national Conquer Chiari Patient Registry database. Neurol Sci 2015;36:1617-1624.

12 Caykoylu A, Ekinci O, Albayrak Y, Kuloglu M, Deniz O: Arnold-Chiari I malformation association with generalized anxiety disorder: a case report. Prog Neuropsychopharmacol Biol Psychiatry 2008;32:1613-1614.
13 Chisholm BT, Velamoor R, Chandarana PC, Cochrane DK: Anxiety disorder in a case of Arnold-Chiari malformation. J Psychiatry Neurosci 1993;18:67-68.

14 Kuloğlu M, Çayköylü A, Ekinci O, Albayrak Y, Deniz O: Comorbid panic disorder and Chiari I malformation: a case report. Yeni Symposium 2009;47:120-122.

15 Del Casale A, Serata D, Rapinesi C, Simonetti A, Tamorri SM, Comparelli A, et al: Psychosis risk syndrome comorbid with panic attack disorder in a cannabis-abusing patient affected by Arnold-Chiari malformation type I. Gen Hosp Psychiatry 2012;34:702.e5-e7.

16 Valentini L, Visintini S, Saletti V, Chiapparini L, Estienne M, Solero CL: Treatment for Chiari 1 malformation (CIM): analysis of a pediatric surgical series. Neurol Sci 2011;32(suppl 3):S321-S324.

17 Schmahmann JD, Sherman JC: The cerebellar cognitive affective syndrome. Brain 1998;121: 561-579.

18 Phillips JR, Hewedi DH, Eissa AM, Moustafa AA: The cerebellum and psychiatric disorders. Front Public Health 2015;3:66.

19 Nosarti C, Reichenberg A, Murray RM, Cnattingius S, Lambe MP, Yin L, et al: Preterm birth and psychiatric disorders in young adult life. Arch Gen Psychiatry 2012;69:E1-E8.

20 Russ SA, Larson K, Halfon N: A national profile of childhood epilepsy and seizure disorder. Pediatrics 2012;129:256-264. 
21 Polanczyk GV, Salum GA, Sugaya LS, Caye A, Rohde LA: Annual research review: a metaanalysis of the worldwide prevalence of mental disorders in children and adolescents. J Child Psychol Psychiatry 2015;56:345-365.

22 American Psychiatric Association: Diagnostic and Statistical Manual of Mental Disorders. Washington, American Psychiatric Association, 2013.

23 Cook JA, Mueser KT: Improving services for parents with psychiatric disabilities: three new opportunities in the field of psychiatric rehabilitation. Psychiatr Rehabil J 2014;37: $1-3$.
24 Lawrence EJ, Rubia K, Murray RM, McGuire PK, Walshe M, Allin M, et al: The neural basis of response inhibition and attention allocation as mediated by gestational age. Hum Brain Mapp 2009;30:1038-1050.

25 Nosarti C, Froudist-Walsh S: Alterations in development of hippocampal and cortical memory mechanisms following very preterm birth. Dev Med Child Neurol 2016;58:35-45.

26 Vohr B: Long-term outcomes of moderately preterm, late preterm, and early term infants. Clin Perinatol 2013;40:739-751.

27 Brossard-Racine M, Limperopoulos C: Normal cerebellar development by qualitative and quantitative MR imaging: from the fetus to the adolescent. Neuroimaging Clin N Am 2016;26:331-339.
28 Poretti A, Boltshauser E, Huisman TA: Prenatal cerebellar disruptions: neuroimaging spectrum of findings in correlation with likely mechanisms and etiologies of injury. Neuroimaging Clin N Am 2016;26:359-372.

29 Fields RD: White matter in learning, cognition and psychiatric disorders. Trends Neurosci 2008;31:361-370.

30 Allen PA, Houston JR, Pollock JW, Buzzelli C, Li X, Harrington AK, et al: Task-specific and general cognitive effects in Chiari malformation type I. PLoS One 2014;9:e94844. 